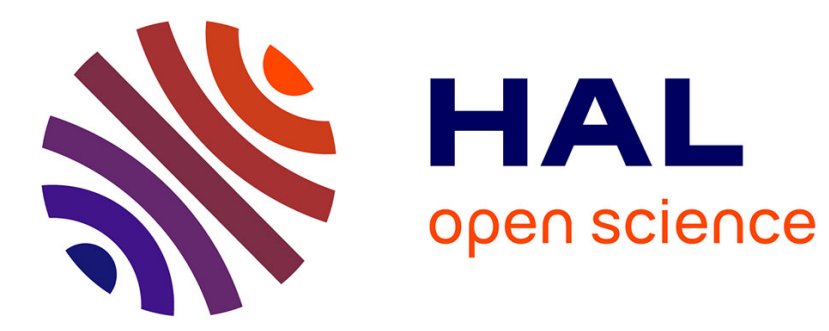

\title{
Dielectrophoresis-assisted creation of cell aggregates under flow conditions using planar electrodes
}

Jonathan Cottet, Alexandre Kehren, Soufian Lasli, Harald van Lintel, François Buret, Marie Frénéa-Robin, Philippe Renaud

\section{- To cite this version:}

Jonathan Cottet, Alexandre Kehren, Soufian Lasli, Harald van Lintel, François Buret, et al.. Dielectrophoresis-assisted creation of cell aggregates under flow conditions using planar electrodes. Electrophoresis, 2019, 10.1002/elps.201800435 . hal-02052550

\section{HAL Id: hal-02052550 https://hal.science/hal-02052550}

Submitted on 19 Jan 2022

HAL is a multi-disciplinary open access archive for the deposit and dissemination of scientific research documents, whether they are published or not. The documents may come from teaching and research institutions in France or abroad, or from public or private research centers.
L'archive ouverte pluridisciplinaire HAL, est destinée au dépôt et à la diffusion de documents scientifiques de niveau recherche, publiés ou non, émanant des établissements d'enseignement et de recherche français ou étrangers, des laboratoires publics ou privés. 


\title{
Dielectrophoresis-assisted creation of cell aggregates under flow conditions using planar electrodes
}

\author{
Jonathan Cottet ${ }^{\mathrm{a}, \mathrm{b}}$, Alexandre Kehren ${ }^{\mathrm{b}}$, Soufian Lasli ${ }^{\mathrm{b}}$, Harald van Lintel ${ }^{\mathrm{b}}$, \\ François Buret ${ }^{\mathrm{a}}$, Marie Frénéa-Robin ${ }^{\mathrm{a}}$ and Philippe Renaud ${ }^{\mathrm{b}}$ \\ a Univ Lyon, Ecole Centrale de Lyon, Université Claude Bernard Lyon 1, INSA Lyon, CNRS, Ampère, F-69130, Ecully, \\ France \\ b École Polytechnique Fédérale de Lausanne, EPFL-STI-IMT-LMIS4, Station 17, CH-1015 Lausanne, Switzerland \\ Corresponding author: Jonathan Cottet \\ Postal address: EPFL - STI - IMT - LMIS4, BM 3125 Station 17, CH - 1015 Lausanne, Switzerland \\ e-mail: jonathan.cottet@epfl.ch; (D http://orcid.org/0000-0001-9377-9823
}

Abbreviations

$\mathrm{AC}$

Alternating Current

BSA

Bovine Serum Albumin

$\mathrm{CM}(\mathrm{f})$

Clausius-Mossotti factor

DC

Direct Current

DEP

Dielectrophoresis

DIW

Deionized Water

DMEM

Dulbecco Modified Eagle Medium

FBS

Fetal Bovine Serum

Received: 10 15, 2018; Revised: 01 20, 2019; Accepted: 01 30, 2019

This article has been accepted for publication and undergone full peer review but has not been through the copyediting, typesetting, pagination and proofreading process, which may lead to differences between this version and the Version of Record. Please cite this article as doi: 10.1002/elps.201800435.

This article is protected by copyright. All rights reserved. 
HEK

IBE

IS

nDEP

PBS

PCB

pDEP

PDMS

PS

$\mathrm{SOI}$
Human Embryonic Kidney

Ion Beam Etching

Impedance Spectroscopy

Negative Dielectrophoresis

Phosphate-Buffered Saline

Printed Circuit Board

Positive Dielectrophoresis

Polydimethylsiloxane

Penicillin Streptomycin

Silicon-On-Insulator

Keywords: Bottom-up assembly, Cell trapping, Dielectrophoresis, Microfluidics

\section{Abstract}

We present a microfluidic platform allowing dielectrophoresis-assisted formation of cell aggregates of controlled size and composition under flow conditions. When specific experimental conditions are met, negative dielectrophoresis (nDEP) allows efficient concentration of cells towards electric field minima and subsequent aggregation. This bottom-up assembly strategy offers several advantages with respect to the targeted application: first, DEP offers precise control of spatial cell organization, which can be adjusted by optimizing electrode design. Then, it could contribute to accelerate the establishment of cell-cell interactions by favoring close contact between neighboring cells. The trapping geometry of our chip is composed of 8 electrodes arranged in a circle. Several parameters have been tested in simulations to find the best configurations for trapping in flow. Those configurations have been tested experimentally with both polystyrene beads and HEK cells. The final design and experimental setup have been optimized to trap cells and release the created aggregates on demand.

\section{Color online: See article online to view Figs. $1-5$ in color.}

Additional supporting information may be found in the online version of this article at the publisher's website.

This article is protected by copyright. All rights reserved. 


\section{Introduction}

Cell aggregates are useful intermediary models between the single cell and the tissue to understand how electric properties are modified in a multicellular construct. Such information can help to improve electrochemotherapy, the use of electric fields to permeabilize cells to enhance the delivery of chemotherapeutic agents directly into cancer cells in vivo [1, 2]. Since tumors are composed of several cells and are surrounded by a 3D environment, the electroporation parameters (e.g. pulse duration and intensity, number of pulses, etc.) need to be adjusted to reach the permeabilization of all the cells in the tumor. In particular, they should take into account the specificity of the treated tumor (size, degree of vascularization, fibrosis, and necrosis); otherwise the treatment might result in suboptimal transfer of the material (gene, drug) in the cell $[3,4]$. While in vitro studies performed on isolated cells provide useful information on how field pulses induce biological membrane permeabilization, more realistic 3D in vitro models are required to mimic the behavior of groups of cells in a tumor or a tissue. Such multicellular constructs can be used as simple models of tumor microregions for the optimization of electropulsation protocols [5], but to this intent it is highly desirable to electrically characterize them.

Most attempts to simulate the dielectric properties of cell assemblies are unconvincing due to the difficulty to model the hierarchical organization of biological tissues in all its complexity. Often biological cells tend to form aggregates of various shapes. While computing the complex permittivity of a cell suspension is relatively feasible, calculating the complex permittivity of touching particles is however not straightforward. Most of the time an aggregate is modeled as an equivalent elementary particle with a specific shape (commonly a sphere or an ellipsoid) and similar electrical properties [6]. This model has been successfully used by Raicu et al. [7] to approximate the organization of the liver cells into hepatic plates. They also showed [6] that in the specific case of spherical aggregates no differences in the analytical model is seen between a suspension of spherical aggregates and particles individually dispersed. However, if the aggregate is composed of polarized cells interacting electrically with each other or physically connected, it is difficult to obtain an analytical model.

This article is protected by copyright. All rights reserved. 
Numerical modeling can be used to calculate the electrical potential distribution inside the aggregate and can provide a more accurate solution to obtain the effective complex permittivity of the aggregate. The mitosis phenomenon has also been simulated by Asami [8] as well as the gap junctions in liver [9] and heart [10]. However, modeling cells in close proximity or in contact through a narrow neck or gap junctions is difficult to perform and requires a lot of computing power. The development of experimental approaches helping to better understand the relationship between single cell and cell assembly properties is therefore of great interest.

Nowadays several methods exist to create experimentally cell aggregates and they can be classified in two categories: contact and non-contact based methods [11]. Contact methods rely on the direct contact with a surface. In the case of non-contact methods cells can be moved with an additional force. Aggregates such as spheroids are often formed with the hanging drop technique where cells in pipetted droplets are concentrated by gravity at the liquid-air interface when the plate is inverted [12]. The properties of this formed aggregate are however not fully controlled and depend on the initial volume of liquid and cells dispensed in the drop. More recently the non-contact methods have become increasingly popular since the force used can offer more control in the formation of the aggregates. Such methods are relying on magnetophoresis [13], acoustophoresis [14], optical trapping [15] and dielectrophoresis. Electric field-based methods present many advantages due to their label-free nature. They can be used both to form cell assemblies and to characterize them using dielectrophoresis and electrical impedance spectroscopy, respectively. The physical and dielectric parameters of the cells can be used to move them. Originally observed with particles [16], dielectrophoresis is commonly used to separate various cells as they present different dielectric properties [17, 18]. Depending on the dielectric properties of the cells and the suspending medium, cells can be directed towards areas of maximum field intensity (which is called positive Dielectrophoresis or pDEP) or repelled in the area of low electric field intensity (which is referred to as negative Dielectrophoresis or nDEP).

Many designs for trapping single cells and cell aggregates have been developed as reviewed by Voldman $[19,20]$. In the case of cell aggregates created by PDEP, the media used are often of low conductivity (in the

This article is protected by copyright. All rights reserved. 
order of the $\mathrm{mS} / \mathrm{m}$ ) to allow to work in the pDEP regime at low frequencies and to reduce the effect of the Joule heating. Most of the electrode geometries used are castellated interdigitated electrodes [21]. Cells are injected in the chip at the beginning and a very small or zero flow is maintained during the trapping process. Cells are often organized as "pearl chains" as described by Jones [22]. Cell culture can be performed afterwards, once the field is stopped, by replacing the medium with fresh culture medium like DMEM with a conductivity in the order of $1 \mathrm{~S} / \mathrm{m}$. In this case the position control is lost.

In the case of cell aggregates created with nDEP, the medium used can be more conductive (in the $\mathrm{S} / \mathrm{m}$ range) since cells will be far from the electrode. However lower conductivity media are often recommended [23]. The most used configurations are quadripolar and octopolar electrodes [24]. Cells can be injected in a microchannel and trapped directly in flow. This solution provides the possibility to continuously add cells to the formed aggregate. More often cells are first injected in the microchannel and then the field is turned on in a second step. Voldman et al. [25] used this methodology to study the efficacy of a DEP trap for single cells. Jaeger et al. [26] later proved that it was possible to trap a single yeast cell in a microchannel by nDEP and let it divide over a period of several hours. However, fabrication of such structures is challenging since it requires the alignment of two sets of electrodes located on two different glass slides separated by a polymer spacer.

Creating cell aggregates that are controllable in size was proposed by Medoro et al [27] where, using an array of individually addressable transistors, cells can be trapped and later contacted to form larger cell aggregates. This method, however, relies on a complex design requiring thousands of transistors and is not used under flow conditions. More recently Menad et al. [28] proposed a design using a network of quadripolar electrodes to form large cell aggregates $(150 \times 150 \times 50 \mu \mathrm{m})$ in a low conductive medium. They noticed that cells will not separate if the field is applied for more than 15 minutes. This system allows to collectively create aggregates regular in size and shape but does not offer a precise control of the number of cells constituting each aggregate.

In the literature, most DEP traps are designed either for single cells or for an uncontrolled number of cells related to the concentration of particles in the initial solution. Creating cell aggregates that are controllable in

This article is protected by copyright. All rights reserved. 
size and number of cells under flow conditions has not been achieved with the designs presented in the literature.

To reach such a performance, the created device should fulfill the following requirements:

- The cells should arrive with the liquid and be captured in a precise position while the flow is maintained during the arrival of the different cells.

- The trap should be activated from the beginning and accept new cells to join the cells already present.

- The size of the trapping area should be adapted to the size of the envisioned aggregate.

- The device should offer the possibility to create aggregates made of different cells in a controlled way.

- The stability of the trap should be robust to flow variations.

- The fabrication process should be relatively simple.

- The applied voltage should be compatible with cell handling.

- The temperature in the chamber should be compatible with cells.

- The device should integrate a sensor to characterize single cells and cell aggregates.

In this paper we propose an electrode trapping geometry that allows cells to be trapped in flow conditions. Based on simulation and experimental results of a test design, an improved design was proposed and validated for the creation of aggregates of controlled size. The full fabricated chip, composed of a PDMS microchannel bonded on a glass substrate with platinum electrodes, integrates both the trapping and the sensing electrodes. The present paper is focused on the optimization of the trapping with dielectrophoresis.

\section{Theory}

The term "dielectrophoresis", as originally introduced by Pohl [16], describes the motion of dielectric particles due to their interaction with a non-uniform electric field.

This article is protected by copyright. All rights reserved. 
The time average dielectrophoretic force acting on a spherical dielectric particle in a suspension medium is

$$
\mathbf{F}_{\mathbf{D E P}}=2 \pi \varepsilon_{m} \varepsilon_{0} r_{e x t}^{3} \operatorname{Re}[C M(f)] \nabla E_{R M S}^{2}
$$

where $\mathrm{E}$ is the electric field, $r_{\text {ext }}$ the external radius of the particle and $C M(f)$ the Clausius-Mossotti factor:

$$
C M(f)=\frac{\varepsilon_{p}^{*}-\varepsilon_{m}^{*}}{\varepsilon_{p}^{*}+2 \varepsilon_{m}^{*}}
$$

$\varepsilon_{p}^{*}$ and $\varepsilon_{m}^{*}$ refer to the complex permittivities of particle and medium, which depend on their respective conductivities and permittivities and on the field angular frequency $\omega$ :

$$
\varepsilon_{i}^{*}=\varepsilon_{i} \varepsilon_{0}-j \frac{\sigma_{i}}{\omega}
$$

where $\varepsilon_{i}$ is the relative permittivity, $\varepsilon_{0}$ the vacuum permittivity, $\sigma_{i}$ the electrical conductivity and $\omega=2 \pi f$ with $f$ the frequency.

For a cell modeled with the "single-shell" structure, which consists of a cytoplasm surrounded by a thin membrane, the equivalent complex permittivity [29] is:

$$
\epsilon_{e q}^{*}=\epsilon_{c m}^{*} \frac{\left(\frac{r_{e x t}}{r_{e x t}-t h_{c m}}\right)^{3}+2\left(\frac{\epsilon_{c p}^{*}-\epsilon_{c m}^{*}}{\epsilon_{c p}^{*}+2 \epsilon_{c m}^{*}}\right)}{\left(\frac{r_{e x t}}{r_{e x t}-t h_{c m}}\right)^{3}-\left(\frac{\epsilon_{c p}^{*}-\epsilon_{c m}^{*}}{\epsilon_{c p}^{*}+2 \epsilon_{c m}^{*}}\right)}
$$

where $t h_{c m}$ is the thickness of the cell membrane, $\varepsilon_{c m}^{*}$ and $\varepsilon_{c p}^{*}$ are respectively the complex cell membrane permittivities of the cell membrane and the cytoplasm. As for a cell $t h_{c m}<<r_{\text {ext }}$, this formula can be approximated as [30]:

$$
\epsilon_{e q}^{*}=\frac{r_{e x t} C_{c m}^{*} \epsilon_{c p}^{*}}{r_{e x t} C_{c m}^{*}+\epsilon_{c p}^{*}}
$$

with

$$
C_{c m}^{*}=C_{c m}-j \frac{G_{c m}}{\omega}
$$

This article is protected by copyright. All rights reserved. 
where $C_{c m}=\epsilon_{c m} / t h_{c m}$ and $G_{c m}=\sigma_{c m} / t h_{c m}$ are the membrane specific capacitance and conductance respectively. The full development can be found in the supplementary material.

Using equations $(1-6)$, the dielectrophoretic behaviour of particles and cells can be predicted. The force depends on the gradient of the squared electric field intensity and exists only in the presence of a non-uniform electric field. It is proportional to the volume of the particle, as well as to the real part of the Clausius-Mossotti factor, $\operatorname{Re}[C M(f)]$. This term, reflecting the polarizability contrast between the particle and its immersion medium, also determines the direction of the force. The sign of $\operatorname{Re}[C M(f)]$ determines the direction of the force. The cases where $(\operatorname{Re}[C M(f)]>0)$ and $(\operatorname{Re}[C M(f)]<0)$ correspond to pDEP and nDEP, respectively (force directed towards or away from maximum field regions).

To trap particles, the dielectrophoretic force should compensate the drag force exerted on each particle by the surrounding fluid. The expression of the drag force (Stokes) is:

$$
\boldsymbol{F}_{\text {Drag }}=6 \pi r_{\text {ext }} \eta \mathbf{v}
$$

with $\eta$ the viscosity of the medium and $\mathbf{v}$ the fluid velocity relative to the particle.

\section{Materials and Methods}

\subsection{Comsol simulations}

The modeling of cell and particle trapping was performed using COMSOL Multiphysics 5.3 and the add-on AC/DC and Particle Tracing modules. Simulated particles were configured as single-shell particles (composed of a cytoplasm surrounded by a thin membrane with HEK cell properties from the literature $\left(r_{e x t}=7.5 \mu m, \sigma_{c p}=\right.$ $0.533 \mathrm{~S} / \mathrm{m}, \varepsilon_{c p}=71, G_{c m}=0 \mathrm{~S} / \mathrm{m}^{2}$ and $C_{c m}=8.5 \mathrm{mF} / \mathrm{m}^{2}$ ) [31]. The equation used to implement the singleshell model with $G_{c m}$ and $C_{c m}$ in COMSOL Multiphysics are presented in the supplementary material. The $A C$ signal applied to each electrode was a sine wave of amplitude $10 \mathrm{~V}_{\mathrm{p} \text { (peak) }}$ and frequency $10 \mathrm{kHz}$. For all designs the flow rate was set to $500 \mu \mathrm{m} / \mathrm{s}$ and the particles were released from the inlet located on the left of each design.

This article is protected by copyright. All rights reserved. 


\subsection{Fabrication}

All chips were fabricated in the cleanroom of the EPFL Center of Microtechnology (CMi) and are made of $20 \mathrm{~nm}$ of titanium/200 $\mathrm{nm}$ of platinum sputtered on float glass and patterned through photolithography and Ion Beam Etching (IBE) for the electrode layer. The microfluidic layer was made out of PDMS casted on a SOI mold with a $50 \mu \mathrm{m}$ high structure. The hydrodynamic resistance of the microchannel was adjusted using a 40 $\mu \mathrm{m}$ wide, $50 \mu \mathrm{m}$ high and $42 \mathrm{~mm}$ long serpentine to reduce the impact of pressure variations. The PDMS slabs were separated and access holes punched. They were later aligned with the electrodes using a mask aligner. For more details on the fabrication process, see [32].

\subsection{Beads preparation}

Solutions of polystyrene beads of 8 and $15 \mu \mathrm{m}$ diameters were prepared from a bead stock solution of known concentration from Sigma Aldrich. The bead stock solution was first diluted ten times in Phosphate Buffer Saline (PBS) to reach a desired concentration of 1000 beads/ $\mu$ l. The PBS was than diluted ten times in Deionized Water (DIW) to reach an electrical conductivity to $0.156 \mathrm{~S} / \mathrm{m}$. This diluted solution had a final concentration of 100 beads $/ \mu$ l. Finally, $0.05 \%$ Tween was added to prevent beads to stick to PDMS.

\subsection{Cell culture and preparation}

Experiments were performed using the Human Embryonic Kidney (HEK) cell line from ATCC. Cells were cultured in a T75 flask using Dulbecco Modified Eagle Medium (DMEM - $\sigma_{m}=1.5 \mathrm{~S} / \mathrm{m}$ ) with $1 \%$ Penicillin Streptomycin (PS) and 10\% Fetal Bovine Serum (FBS). Cells were collected twice per week and re-suspended when a confluency of about $80 \%$ was reached.

Experimental medium consisted of DMEM diluted ten times in deionized water to reach $\sigma_{m}=0.156 \mathrm{~S} / \mathrm{m}$ in order to reduce thermal heating by Joule effect. The osmolarity of the solution was corrected to reach the physiological value of $300 \mathrm{mOsm} / \mathrm{L}$ by addition of dextrose. Both the electrical conductivity and osmolarity were experimentally verified with a conductivity meter (InLab 710 from Mettler Toledo) and a microosmometer (Fiske Model 210).

This article is protected by copyright. All rights reserved. 


\subsection{BSA preparation}

A 10\% Bovine Serum Albumin (BSA) stock solution was prepared by dissolving $100 \mathrm{mg}$ of BSA lyophilized powder (Sigma Aldrich) in $1 \mathrm{ml}$ of deionized water and by gently rocking the capped tube until the BSA was fully dissolved.

\subsection{Experimental setup}

The microfluidic chip is placed on a Printed Circuit Board (PCB), which enables the connection of DEPtrapping and DEP-centering electrodes to two arbitrary function generators (HMF2525 from Rohde \& Schwarz), and also detection electrodes (not discussed in this article). The PCB, presented in Figure S6A in the supplementary material, is mounted on an inverted microscope (from DMIL Leica), and a uEye camera (UI3060CP Rev. 2 from IDS Imaging Development Systems GmbH) enables the real time visualization with the computer. Fluid was automatically injected in the chip using either a flow control pump (Nemesys Syringe Pump) or a pressure controller (Flow EZ from Fluigent). The flow control was used to validate the trapping at a specific flow rate and the pressure control to reduce the flow variation and the flow rate. An impedance counting unit, presented in [33], was placed before and after the trapping chamber. Two flow controllers Flow EZ from Fluigent were used temporarily one after the other: a 1000 mbar to fill the chip with liquid and a 25 mbar for precise flow control. Applied frequencies were $f_{D E P}=10 \mathrm{kHz}$ for DEP centering and trapping electrodes. The schematics of the experimental setup with pressure control is presented in Figure S6B in the supplementary material. The PDMS chip was first filled with deionized water at 100 mbar and then primed by injecting the BSA solution for 10 minutes at 10 mbar to prevent the adhesion of cells to the PDMS walls and to the glass. The cell suspension was then injected at 25 mbar before reducing the pressure to 2 mbar for the trapping.

This article is protected by copyright. All rights reserved. 


\section{Results}

\subsection{Trapping parameters}

The choice of a given electrode configuration (size, shape, location relative to the microchannel, etc) is obviously a critical determinant of trapping efficiency. Then, for a given configuration, 4 parameters can be adjusted:

- The electric conductivity of the medium $\sigma_{m}$

- $\quad$ The trapping frequency of the electric field $f_{D E P}$

- The voltage applied on the electrode $V$

- $\quad$ The flow speed $v$ (and flow rate $Q$ )

For both polystyrene beads and HEK cells, trapping with nDEP with the lowest possible voltage requires the real part of the Clausius-Mossotti to be around -0.5 (maximum negative achievable amplitude for a spherical particle). This can be performed in highly conductive medium [34, 35] but might lead to Joule heating and electrothermal flow motion [23]. Working with a lower electrical conductivity will reduce the Joule heating while keeping the same dielectrophoretic response at low frequencies. Polystyrene beads and HEK cells dielectrophoretic behavior were predicted in PBS $\left(\sigma_{m}=1.5 \mathrm{~S} / \mathrm{m}\right)$ and 10X PBS $\left(\sigma_{m}=0.156 \mathrm{~S} / \mathrm{m}\right)$.

As can be seen on the plots of $\operatorname{Re}[C M(f)]$ (Figures S1 and S2 in the supplementary material obtained using the MyDEP software [36]), up to $10 \mathrm{kHz}$ the dielectrophoretic force will be maximal. Thus $\sigma_{m}=$ $0.156 \mathrm{~S} / \mathrm{m}$ was selected as well as $f_{D E P}=10 \mathrm{kHz}$. An initial flow speed of $500 \mu \mathrm{m} / \mathrm{s}$ was set for the simulation. Reducing the flow rate will enable to reduce the amplitude of the electric field but if this value is too low, particles will sediment in the microchannel and will not reach the trapping chamber. On the contrary, a high flow rate will require a high voltage on the electrode which may lead to overheating of the suspension medium.

This article is protected by copyright. All rights reserved. 


\subsection{Trapping simulation and test design}

The test design used to investigate the trapping of particles and cells is composed of 8 electrodes arranged in a circle as presented in Figure 1A. Each electrode is $100 \mu \mathrm{m}$ long and $50 \mu \mathrm{m}$ wide. The fluidic inlet and outlet are respectively located at electrodes 1 and 5. A recess of $50 \mu \mathrm{m}$ separate each electrode from the trapping chamber. The electrodes are using the so-called "liquid electrodes" principle [37]. In this configuration, coplanar electrodes are positioned at the bottom of dead-end chambers. This creates a homogeneous electric field over the channel height if the distance of the electrodes to the channel is at least equal to the channel height [38].

The test design offers a high flexibility to evaluate different configurations of the electrodes to trap particles and cells with dielectrophoresis. Various combinations of those 8 electrodes were simulated in Comsol with the particle tracing module. This first set of simulations offers the possibility to evaluate qualitatively the trapping and to compare the different trapping configurations. The fluid velocity profile is presented in Figure 1B. The flow profiles corresponding to each dashed line are presented in Figure S3 in the supplementary materials. With a sinusoidal trapping voltage $V_{D E P}=10 V_{p}$ at $10 \mathrm{kHz}, 5$ configurations enabled the trapping of $15 \mu \mathrm{m}$ cells with HEK properties in the simulation. It is important to notice that trapping with two active electrodes ( 4 at $+V$ and 6 at $-V$ ) or four active electrodes ( $3 / 4$ at $+V$ and $6 / 7$ at $-V$ ) does not work for this voltage. The particles get centered in the trapping chamber and slow down but do not get trapped. Some configurations, for example one with 3 active electrodes ( 5 at $+\mathrm{V}$ and $2 / 8$ at $-\mathrm{V}$ ), will trap the particles but will direct them in the recess of the unused electrodes (4 and 6). More details are presented in Table S1 and Figure S5 in the supplementary material.

The best trapping configuration is the one that offers the highest DEP force on the particle to compensate the drag force. This configuration corresponds to the configuration displayed in Figure $2 \mathrm{~A}$ and $2 \mathrm{~B}$ with 3 active electrodes (5 at $+\mathrm{V}$ and $4 / 6$ at $-\mathrm{V}$ ). Since the DEP force is linked to $\nabla E_{R M S}^{2}$, the $\mathrm{x}$ component of the DEP force, proportional to $\frac{\partial \mathbf{E}^{2}}{\partial x}$, represented by the surface in Figure 2A, should overcome the drag force to allow cell trapping.

This article is protected by copyright. All rights reserved. 
In this case the particles should be stopped at approximately $24 \mu \mathrm{m}$ of the center of the chamber when the resulting force (black dashed line) is equal to zero as shown in Figure 2B, marked by the red vertical dashed line $x_{\text {trap }}$. A 3D representation of the amplitudes of the DEP and drag force is presented in Figure 2C and 2D. The intersection between the two surfaces corresponds to a resulting force equal to zero. In the chip the particle will first reach the $x$ trapping line materialized by the white/red dashed line in Figure 2D.

Figure $2 \mathrm{E}$ to $2 \mathrm{H}$ present the evolution of the $\mathrm{y}$ component of the DEP and drag forces for the chosen configuration of the electrodes. At $\mathrm{x}=0$, as displayed in Figure $2 \mathrm{E}$, the $\mathrm{y}$ component of the drag force is much smaller than the $y$ component of the DEP force. In this case, the particle would be exposed to a resulting force pushing it far from the center depending on its initial lateral position, either toward electrode 7 (zone 1) or electrode 3 (zone 2).

At the trapping position $\mathrm{x}=\mathrm{x}_{\text {trap }}$ presented in Figure $2 \mathrm{~F}$, both the DEP and drag forces push the particle towards the center of the microchannel in a stable trapping position (zones 2 and 3). Zone 2 and 3 are delimited by two other dashed lines which would correspond to two other equilibrium positions located at $\pm 64 \mu \mathrm{m})$. However, those two other equilibrium positions are unstable since a particle located farther than 50 $\mu \mathrm{m}$ from the center will be pushed either towards electrode 7 (zone 1) or electrode 3 (zone 4). Figure $2 \mathrm{G}$ and $2 \mathrm{H}$ present a 3D representation of the amplitude of the $y$ component of the two forces.

The amplitude of the $y$ component is two orders of magnitude smaller than the $x$ component. In all cases, the particles will first reach the trapping position due to the $\mathrm{x}$ component of the force (white/red dashed line in Figure 2D and $2 \mathrm{H}$ ) and then get centered in the microchannel due to the y component as illustrated in Figure $2 \mathrm{H}$ with the grey particle and the arrow until it reaches the black cross.

The trapping positions are also called holding points [25] and correspond to the positions where both the $x$ and $y$ components of the resulting force are equal to zero. This equilibrium position should be stable as long as the flow rate is constant.

This article is protected by copyright. All rights reserved. 


\subsection{Experimental testing of the test design}

First experiments were performed using $8 \mu \mathrm{m}$ diameter beads with a flow controller (syringe pump) with the configuration presented in Figure 2 for $V_{D E P}=17.3 V_{p}$ at $f_{D E P}=10 \mathrm{kHz}$. The medium conductivity and flow rate were set to $\sigma_{m}=0.156 \mathrm{~S} / \mathrm{m}$ and $Q=1 \mu L / h$, respectively. Polystyrene beads were successfully trapped in the chamber and formed clusters that were moved towards the inlet as the size of the cluster increased. The evolution of the cluster position followed the profile of the simulation-predicted position of a particle of increasing size. After stopping the DEP trapping voltage, the particles composing the cluster separated from each other.

This approach was repeated successfully for HEK cells, switching from a flow controller to a pressure controller to enable to reduce the flow rate and to have a better control at small flow rates. Trapping was achieved for the lowest possible voltage of $V_{D E P}=22.5 V_{p}$ and a pressure $P_{i n}=2 \mathrm{mbar}$ as presented in Figure 3. This time a group of cells could be formed in the chamber as more cells arrived in the chamber. The video showing aggregate formation is available in the supplementary material [39].

\subsection{Discussion on the test design}

Cells were successfully trapped in the chamber and formed a small aggregate. Quantification of the time needed to observe adhesion between the cells requires to overcome certain inherent limitations of the design. Out of the 8 electrodes only 3 were used to perform the trapping. However large aggregates were not stable due to the fact that the outlet of the chamber corresponds to a narrowing of the channel thus a reacceleration zone for the fluid. In addition, the trapping zone is close to the electrodes, which due to the higher temperature, might lead to cell damage and aggregate disruption. Another limitation of the trapping with this configuration is that cells arrive continuously in the trapping chamber, increasing the size of the aggregate. Reducing the pressure to prevent the arrival of new cells requires to dynamically modify the trapping voltage in order to preserve the same position of the cells in the chamber.

This article is protected by copyright. All rights reserved. 


\section{Design and setup improvement}

Improving the cell trapping requires to improve both the design layout and the experimental setup.

\subsection{Design improvements}

In order to improve the stability of the trapping and to avoid instabilities such as reacceleration in the vicinity of the trapping area, the fluidic design can be modified, based on the previous study, as displayed in Figure 4. Since only 3 electrodes out of 8 were used for trapping, the improved design should only have 3 (electrodes 2, 3 and 4 in Figure 4D). Doubling the electrodes recess, i.e. the distance between the electrode and the chamber, will reduce the heat transfer to the trapping zone. Similarly, doubling the width of the trapping chamber will reduce the fluid velocity hence the particle velocity and drag force. Moving this widening (respectively narrowing of the channel) before the trapping chamber (respectively after) will improve the stability of the trapping since the drag force will be lower and constant in the center of the chamber. To further reduce the fluid velocity in the chamber, additional outlets can be added to the side electrodes (electrodes 2 and 4 in Figure 4D) while ensuring a high hydrodynamic resistance to prevent particles to go through the auxiliary outlets. This can be obtained by having relatively narrow and long microchannels.

For comparison purpose, the improved design presented in Figure 4 was simulated with the same voltage, the same channel height and the same inlet flow speed as the test design presented in Figure 2. Figure $4 \mathrm{~A}$ presents the fluid velocity profile in the improved design. The flow profiles corresponding to each dashed line are presented in Figure S4 in the supplementary material. The field and trapping simulations are presented in Figure $4 \mathrm{~B}$ to $4 \mathrm{~F}$. With this design, the particle should be trapped closer to the center of the trapping chamber (the center, $\mathrm{x}=0$, corresponds to the geometrical center between electrodes 1 and 3 ) at $x_{\text {trap }}=17 \mu \mathrm{m}$ as presented in Figure 4B. Two equilibrium positions, where the resulting force is equal to zero, exist along the $x$ axis as presented in Figure $4 \mathrm{C}$ but only the one located between zones 1 and 2, with the abscise $x_{\text {trap }}=17 \mu \mathrm{m}$, is stable. A 3D representation of the amplitudes of the DEP and drag force is presented in Figure 4D. The intersection between the two surfaces corresponds to a resulting force equal to zero. Figure $4 \mathrm{E}$ and $4 \mathrm{~F}$ present the evolution of the $y$ component of the DEP and drag forces for the chosen configuration of the electrodes. In

This article is protected by copyright. All rights reserved. 
all cases, the particles will first reach the trapping position due to the $\mathrm{x}$ component of the resulting force (white/red dashed line in Figure $4 \mathrm{D}$ and $4 \mathrm{~F}$ ) and then get centered in the microchannel due to the $\mathrm{y}$ component of the resulting force as illustrated in Figure $4 \mathrm{~F}$ until it reaches the white cross.

This design, while improving the trapping, should however not concentrate particles at a specific point as predicted, but in a wider area (making more of a chain) due to the wide zone of low fluid velocity. A $4^{\text {th }}$ electrode (number 1 in Figure 4D) was then added to re-center the cell aggregate and prevent new particles and cells to join the aggregate once the desired number of cells in the aggregate is reached.

\subsection{Setup Improvements}

To be able to prevent new cells from coming in the trapping chamber, the experimental setup was fully controlled with LabVIEW ${ }^{\mathrm{TM}}$ (National Instruments). The experimental setup, presented in the Materials and Methods section and displayed in Figure S6 in the supplementary material, provides a control of all the instruments through a dedicated interface. When the required number of cells in the chamber is reached, the flow can be decreased together with the DEP trapping voltage. Once the arrival of new cells is stopped, electrode 1 from Figure 4D is supplied with the same voltage as electrode 3, sealing the DEP cage and recentering the cell aggregate. Further increase of the voltage can improve the compacting of the aggregate.

\subsection{Experiments and trapping}

Experiments using the fully automated setup and the improved design are presented in Figure 5 . The chip is first filled with deionized water and then primed with BSA as presented in Figure 5A. The suspension of cells is injected and once the cells start to reach the trapping chamber, the pressure is decreased to $2 \mathrm{mbar}$. Cells arrive slowly in the chamber (Figure 5B) forming a cell line (Figure 5 C). Once the desired number of cells is reached, 21 cells in Figure 5D, both DEP trapping voltage and flow rate are reduced to prevent additional cells to reach the trapping zone. Electrode 1, located on the left, is activated leading to the centering of the cell aggregate (Figure 5E). The DEP force is also increased to compact the aggregate. As displayed in Figure 5F, the aggregate is more compact after $5 \mathrm{~min}$. The DEP force is then cancelled and the flow rate increased to help the formed aggregate to leave the trapping chamber as shown in Figure 5G. The aggregate leaves the trapping

This article is protected by copyright. All rights reserved. 
chamber and no cell separation is observed, even in a narrower meander (width $40 \mu \mathrm{m}$ ) located after the trapping chamber as shown in Figure 5H. See supplementary material for the related video [40].

\section{Discussion}

The proposed design offers a new way to create cell aggregates, on chip, with a controlled number of cells. It has been obtained with the help of simulations that required a few simplifications.

Cells simulated with Comsol in the particle tracing module are considered as point particles. It calculates particle positions based on the forces at a specific point and displays the points as particles of specified diameter. Similarly, simulated particles do not disturb the flow leading to some inaccuracy in the simulations related to the laminar flow. Particles can interpenetrate each other hence releasing many particles at the inlet will only lead to particles aggregating at one specific location with their center at this same point. A similar conclusion can be drawn for the wall penetration of particles. A possible improvement of the simulation to correct this effect would be to use particles defined as a material and calculate step by step their position. This would however require using a moving mesh for the particles and a lot of computing power. This computing power should be even bigger if several particles are simulated at the same time. Their interactions should also be modeled for increased accuracy.

Simulations for the first design served as a basis for comparison between several electrode configurations in order to select the most promising one. The required voltage for trapping is higher than that expected from the simulations. These discrepancies can be explained by the fact that for the sake of simplifications, calculations were performed on a $2 \mathrm{D}$ model. In $2 \mathrm{D}$ simulations, electrodes are considered as equipotential lines located on the side of the microchannel. However, those equipotential lines, equivalent to "liquid electrodes" [37] are created by planar electrodes with a higher voltage in the 3D device. Furthermore, since the experiments are performed with pressure control, the flow speed depends on the design and tubing used. Simulating such system would require a 3D model implying lot of computing resources. The best configuration has been tested experimentally and proved to be capable of trapping polystyrene beads as well as HEK cells

This article is protected by copyright. All rights reserved. 
under flow conditions. This first design was however not optimal for the trapping. An improved design was simulated, tested and proved to be capable of trapping the desired number of cells. However, creating a real "cell by cell aggregate" requires cells to be initially sufficiently separated, which is a point that still needs to be improved. As two impedance sensing stages, presented in [33], have been added upstream and downstream the trapping area, it should also be possible to detect pre-aggregated cells and take them into account while evaluating the final aggregate size.

The absolute size limit of the cell assemblies is the size of the trapping chamber in both designs. Additionally, the size of the microchannel $(50 \mu \mathrm{m} \times 50 \mu \mathrm{m})$ can also be a limitation as cell aggregates with a larger cross-section might get stuck in the microchannel. Adding cells to the assembly will lead to two opposite and competitive effects. On the one hand, adding cells will create a larger assembly which will behave as a larger particle which has been confirmed experimentally. On the other hand, adding cells will reduce the crosssection of the microchannel available for the fluid which will lead to a faster flow and thus decrease the trapping. Experimentally, the stability of the trapping has shown to be increased when the number of polystyrene beads forming a cluster increased. This effect can possibly be explained by the dependence of the force on the object size: the drag force is proportional to $r_{\text {ext }}$ while the DEP force is proportional to $r_{\text {ext }}{ }^{3}$.

All cells remained together as a permanent aggregate after 5 min of contact between trapped cells. While cell-cell interactions are favored thanks to dipole-dipole attraction forces between neighboring cells, the nature of the creation of permanent aggregates is not yet fully understood. Possibly cadherin-cadherin interactions take place as suggested by Menad et al. [28]. Cadherins are membrane proteins related to the physical linkages between cells. Mechanical forces such as compression enhance the protein-mediated adhesion as shown by Pontani et al. [41].

The number of cells composing the aggregate is controlled visually by means of a camera. This number could also be directly obtained using impedance flow cytometry. The created aggregate could be studied in a similar way.

This article is protected by copyright. All rights reserved. 
The here presented set-up offers the possibility to further investigate interactions between different cell types by creating composite aggregates. Using different inlets for different cell types will offer the possibility to create composite aggregates with a known composition; using impedance flow cytometry the properties of each cell and of the created aggregates can be determined.

Aggregates created on-chip could also be part of a Lab-on-a-chip by adding the possibility to electroporate the created aggregates and observe their properties before and after electroporation.

\section{Concluding remarks}

In this article, a geometry for trapping particles and cells using coplanar electrodes and PDMS microchannels is presented. Different electrodes combinations have been tested by means of simulation upon which the best candidate was tested experimentally and further improved in terms of geometry and operation. Controlled aggregates can now be created and further studied directly on chip.

\section{Acknowledgements}

The authors would like to thank Margaux Duchamp for the help provided with the cell culture. The authors acknowledge the support from the CMi staff at EPFL for their technical assistance. The Ampere lab would like to acknowledge support from the Institut National de la Santé et de la Recherche Médicale (INSERM, Plan Cancer, Physicancer Program, Dynamo project). The authors also acknowledge the support of the Programme d'Avenir Lyon Saint-Etienne (PALSE mobility grant) and the Laboratoire d'Excellence iMUST (ANR-10-LABX-0064/ANR-11-IDEX-0007) from University of Lyon as well as the Doctoral school 160 EEA of the University of Lyon for the mobility grants allocated.

The authors have declared no conflict of interest.

\section{References}

[1] Breton, M., Mir, L. M., Bioelectromagnetics 2012, 33, 106-123; doi:10.1002/bem.20692.

This article is protected by copyright. All rights reserved. 
[2] Escoffre, J. M., Rols, M. P., Curr. Pharm. Des. 2012, 18, 3406-3415; doi:10.2174/138161212801227087.

[3] Marty, M., Sersa, G., Garbay, J. R., Gehl, J., Collins, C. G., Snoj, M., Billard, V., Geertsen, P. F., Larkin, J. O., Miklavcic, D., Pavlovic, I., Paulin-Kosir, S. M., Cemazar, M., Morsli, N., Soden, D. M., Rudolf, Z., Robert, C., O’Sullivan, G. C., Mir, L. M., Eur. J. Cancer Suppl. 2006, 4, 3-13; doi:10.1016/j.ejcsup.2006.08.002.

[4] Mir, L. M., Gehl, J., Sersa, G., Collins, C. G., Garbay, J.-R., Billard, V., Geertsen, P. F., Rudolf, Z., O'Sullivan, G. C., Marty, M., Eur. J. Cancer Suppl. 2006, 4, 14-25; doi:10.1016/j.ejcsup.2006.08.003.

[5] Chopinet, L., Wasungu, L., Rols, M. P., Int. J. Pharm. 2012, 423, 7-15; doi:10.1016/j.ijpharm.2011.04.054.

[6] Raicu, V., Feldman, Y., Dielectric Relaxation in Biological Systems: Physical Principles, Methods, and Applications, Oxford University Press, Oxford 2015; doi:10.1093/acprof:oso/9780199686513.001.0001.

[7] Raicu, V., Saibara, T., Enzan, H., Irimajiri, A., Bioelectrochem. Bioenerget. 1998, 47, 333-342; doi:10.1016/S0302-4598(98)00172-X.

[8] Asami, K., Gheorghiu, E., Yonezawa, T., Bba-Gen Subjects 1998, 1381, 234-240; doi:10.1016/S0304-4165(98)00033-6.

[9] Gersing, E., Bioelectrochem. Bioenerget. 1998, 45, 145-149; doi:10.1016/s0302-4598(98)00079-8. [10] Schaefer, M., Gross, W., Ackemann, J., Gebhard, M. M., Bioelectrochemistry 2002, 58, 171-180; doi:10.1016/s1567-5394(02)00152-4.

[11] Rodríguez-Dévora, J. I., Shi, Z. D., Xu, T., Biotechnol. J. 2011, 6, 1454-1465; doi:10.1002/biot.201100100.

[12] Asghar, W., El Assal, R., Shafiee, H., Pitteri, S., Paulmurugan, R., Demirci, U., Mater. Today 2015, 18, 539-553; doi:10.1016/j.mattod.2015.05.002.

[13] Souza, G. R., Molina, J. R., Raphael, R. M., Ozawa, M. G., Stark, D. J., Levin, C. S., Bronk, L. F., Ananta, J. S., Mandelin, J., Georgescu, M. M., Bankson, J. A., Gelovani, J. G., Killian, T. C., Arap, W., Pasqualini, R., Nat. Nanotechnol. 2010, 5, 291-296; doi:10.1038/nnano.2010.23.

[14] Guo, F., Mao, Z. M., Chen, Y. C., Xie, Z. W., Lata, J. P., Li, P., Ren, L. Q., Liu, J. Y., Yang, J., Dao, M., Suresh, S., Huang, T. J., Proc. Natl. Acad. Sci. U.S.A. 2016, 113, 1522-1527; doi:10.1073/pnas.1524813113.

[15] Akselrod, G. M., Timp, W., Mirsaidov, U., Zhao, Q., Li, C., Timp, R., Timp, K., Matsudaira, P., Timp, G., Biophys. J. 2006, 91, 3465-3473; doi:10.1529/biophysj.106.084079.

This article is protected by copyright. All rights reserved. 
[16] Pohl, H. A., J. Appl. Phys. 1951, 22, 869-871; doi:10.1063/1.1700065.

[17] Gascoyne, P. R. C., Huang, Y., Pethig, R., Vykoukal, J., Becker, F. F., Meas. Sci. Technol. 1992, 3, 439-445; doi:10.1088/0957-0233/3/5/001.

[18] Fiedler, S., Shirley, S. G., Schnelle, T., Fuhr, G., Anal. Chem. 1998, 70, 1909-1915; doi:10.1021/ac971063b.

[19] Voldman, J., Annu. Rev. Biomed. Eng. 2006, pp. 425-454; doi:10.1146/annurev.bioeng.8.061505.095739.

[20] Voldman, J., BioMEMS and Biomedical Nanotechnology 2007, pp. 159-186; doi:10.1007/978-0387-25845-4_8.

[21] Sebastian, A., Buckle, A. M., Markx, G. H., Biotechnol. Bioeng. 2007, 98, 694-700; doi:10.1002/bit.21416.

[22] Jones, T. B., Electromechanics of Particles, Cambridge University Press, Cambridge, New York 1995; doi:10.1017/cbo9780511574498.

[23] Puttaswamy, S. V., Sivashankar, S., Chen, R. J., Chin, C. K., Chang, H. Y., Liu, C. H., Biotechnol J 2010, 5, 1005-1015; doi:10.1002/biot.201000194.

[24] Markx, G. H., Talary, M. S., Pethig, R., J. Biotechnol. 1994, 32, 29-37; doi:10.1016/01681656(94)90117-1.

[25] Voldman, J., Braff, R. A., Toner, M., Gray, M. L., Schmidt, M. A., Biophys. J. 2001, 80, 531-541; doi:10.1016/S0006-3495(01)76035-3.

[26] Jaeger, M. S., Uhlig, K., Schnelle, T., Mueller, T., J. Phys. D: Appl. Phys. 2008, 41, 175502; doi:10.1088/0022-3727/41/17/175502.

[27] Medoro, G., Manaresi, N., Leonardi, A., Altomare, L., Tartagni, M., Guerrieri, R., IEEE Sens. J. 2003, 3, 317-325; doi:10.1109/Jsen.2003.814648.

[28] Menad, S., Franqueville, L., Haddour, N., Buret, F., Frenea-Robin, M., Acta Biomater. 2015, 17, 107-114; doi:10.1016/j.actbio.2015.01.011.

[29] Irimajiri, A., Hanai, T., Inouye, A., J. Theor. Biol. 1979, 78, 251-269; doi:10.1016/00225193(79)90268-6.

[30] Gascoyne, P. R. C., Becker, F. F., Wang, X. B., Bioelectrochem. Bioenerget. 1995, 36, 115-125; doi:10.1016/0302-4598(94)05015-M.

This article is protected by copyright. All rights reserved. 
[31] Zimmermann, D., Kiesel, M., Terpitz, U., Zhou, A., Reuss, R., Kraus, J., Schenk, W. A., Bamberg, E., Sukhorukov, V. L., J. Membr. Biol. 2008, 221, 107-121; doi:10.1007/s00232-007-9090-4.

[32] Cottet, J., Vaillier, C., Buret, F., Frenea-Robin, M., Renaud, P., Biomicrofluidics 2017, 11, 064111; doi:10.1063/1.5001145.

[33] Cottet, J., Kehren, A., van Lintel, H., Buret, F., Frénéa-Robin, M., Renaud, P., Microfluid. Nanofluid. 2019, 23; doi:10.1007/s10404-018-2178-6.

[34] Fuhr, G., Glasser, H., Muller, T., Schnelle, T., Biochim. Biophys. Acta 1994, 1201, 353-360; doi:10.1016/0304-4165(94)90062-0.

[35] Glasser, H., Fuhr, G., Bioelectrochem. Bioenerget. 1998, 47, 301-310; doi:10.1016/s03024598(98)00146-9.

[36] Cottet, J., Fabregue, O., Buret, F., Renaud, P., Frénéa-Robin, M., 2018; doi:10.1007/s10404-0182178-6.

[37] Demierre, N., Braschler, T., Linderholm, P., Seger, U., van Lintel, H., Renaud, P., Lab Chip 2007, 7, 355-365; doi:10.1039/b612866a.

[38] Demierre, N., PhD Thesis: Continuous-flow separation of cells in a lab-on-a-chip using "liquid electrodes" and multiple-frequency dielectrophoresis, EPFL, Lausanne 2008; doi:10.5075/epfl-thesis4099.

[39] Cottet, J., 2018; doi:10.5281/zenodo.1434999.

[40] Cottet, J., 2018; doi:10.5281/zenodo.1435006.

[41] Pontani, L. L., Jorjadze, I., Viasnoff, V., Brujic, J., Proc Nat/ Acad Sci U S A 2012, 109, 9839-9844; doi:10.1073/pnas.1201499109.

This article is protected by copyright. All rights reserved. 


\section{Figure captions}

Figure 1 A) Trapping design composed of 8 electrodes arranged in circle (dark gray rectangles). The fluidic inlet and outlet are respectively located at electrodes 1 and 5 . All electrodes are separated from the trapping chamber by a $50 \mu \mathrm{m}$ recess. Scalebar $50 \mu \mathrm{m}$. B) COMSOL Multiphysics 2D Simulation of the fluid velocity profile, in $\mu \mathrm{m} / \mathrm{s}$, in the chip with an inlet velocity of $500 \mu \mathrm{m} / \mathrm{s}$.

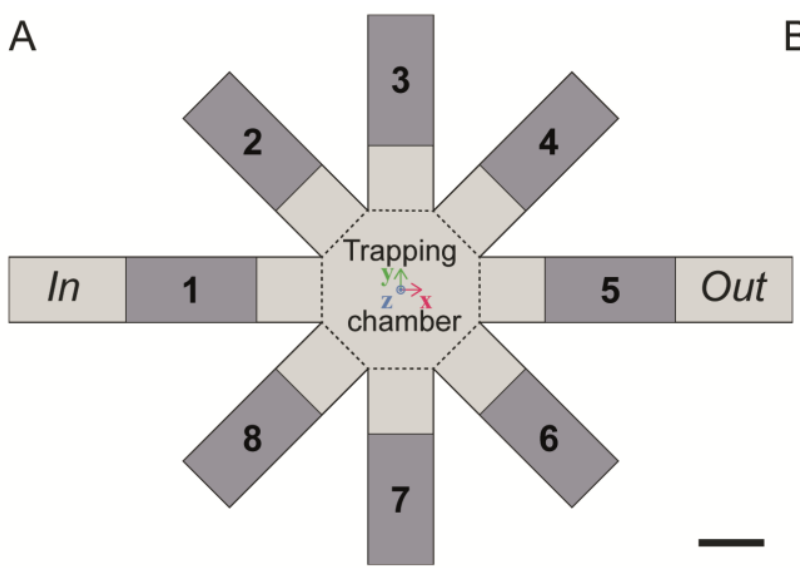

B
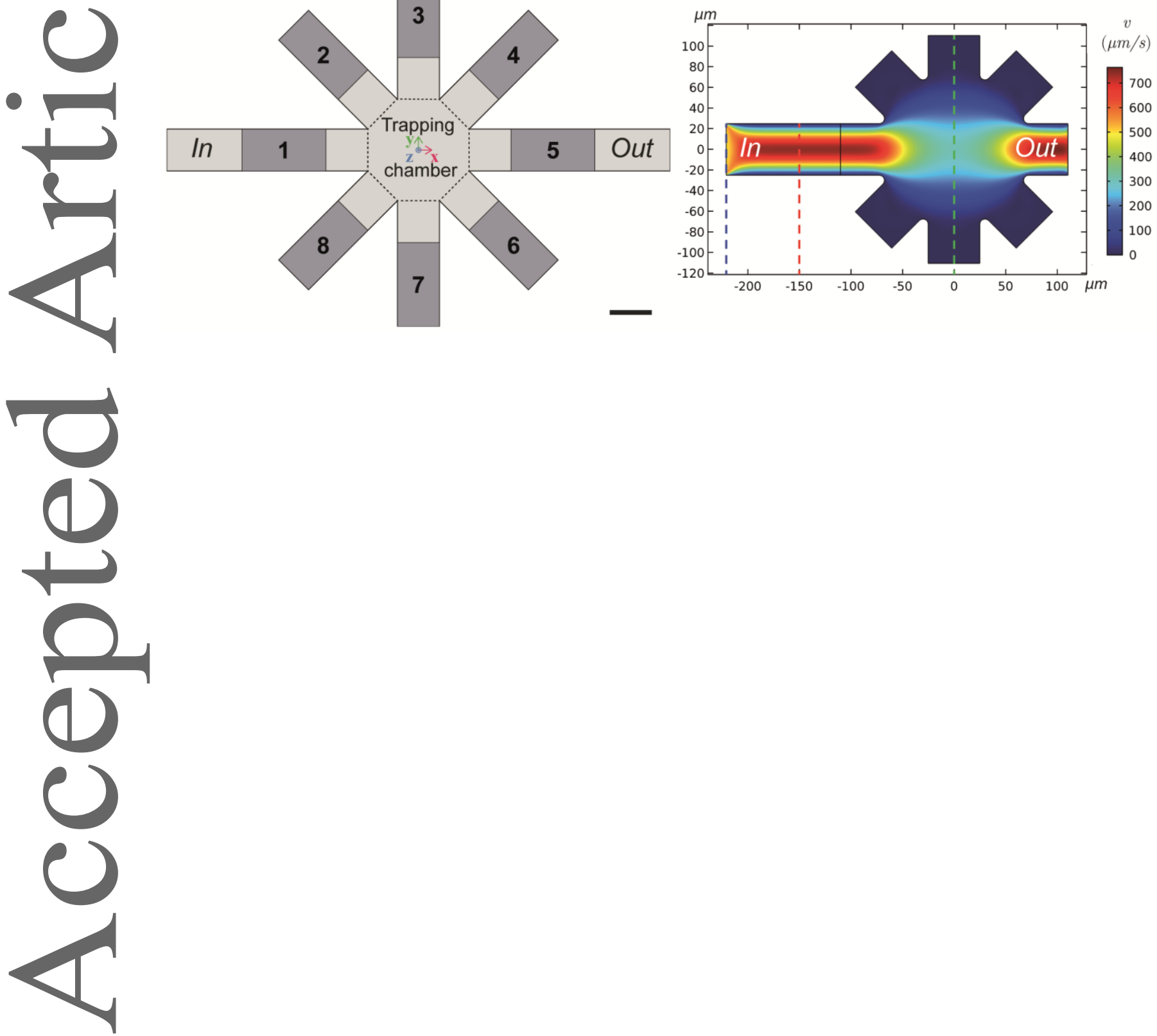

This article is protected by copyright. All rights reserved. 
Figure $2 \mathrm{~A}$ ) 2D simulation of the trapping position of a cell using 3 electrodes $(4,5$ and 6$)$ supplied with $V=10 V_{p}$ at 10 $\mathrm{kHz}$. The surface represents $\frac{\partial E^{2}}{\partial x}$. The white dot indicates the trapping position of a HEK cell (diameter $\left.15 \mu \mathrm{m}\right)$. B) Evolution of the DEP and drag forces along the $A A^{\prime}$ cut. The resulting force is represented with the black dashed line and the red dashed vertical line indicates the position where the resulting force in equal to zero. The gray dot with the arrow indicates the direction of the force on the particle and the red dashed line the trapping position. C) Side and D) Top 3D representations of the amplitudes of the DEP (in blue) and drag (in green) forces along the $x$ axis in the chip. The white/red dashed line in $D$ indicates where the $x$ component of two forces are equal in the trapping chamber. The electrode numbers are indicated. A logarithmic scale is used for both representations. Evolution of the DEP and drag forces along the $y$ axis at $E$ ) $x=0$ and $F$ ) $x=x_{\text {trap }}=24 \mu \mathrm{m}$. The resulting force is represented with the black dotted line and the red dotted line indicates the positions where the resulting force is equal to zero. The gray dot with the arrow indicates the direction of the force on the particle. G) Side and H) Top 3D representations of the amplitudes of the DEP (in blue) and drag (in green) forces along the $y$ axis in the chip. The white/red dashed line in $\mathrm{H}$ indicates where the $\mathrm{x}$ component of two forces are equal (from $\mathrm{D}$ ) and the black cross the final position of the particle. The electrode numbers are indicated. A logarithmic scale is used for both representations.

This article is protected by copyright. All rights reserved. 
Figure 3 Example of HEK cell aggregation in flow conditions using 3 electrodes. The time stamps are in the mm:ss format. Scalebar $50 \mu \mathrm{m}$. A) Chip with only fluid inside. B) Cells reaching the trapping zone are stopped when the DEP force compensates the drag force. C) and D) The aggregate increased in size as more cells were reaching the trapping zone with 5 cells (C) and 8 cells and 1 arriving (D). Channel height $50 \mu \mathrm{m}$, Inlet and Outlet width $50 \mu \mathrm{m}, \mathrm{V}=22.5 V_{p}$ at $f_{D E P}=10 \mathrm{kHz}, \sigma_{m}=0.156 \mathrm{~S} / \mathrm{m}$ and $P_{\text {in }}=2 \mathrm{mbar}$.
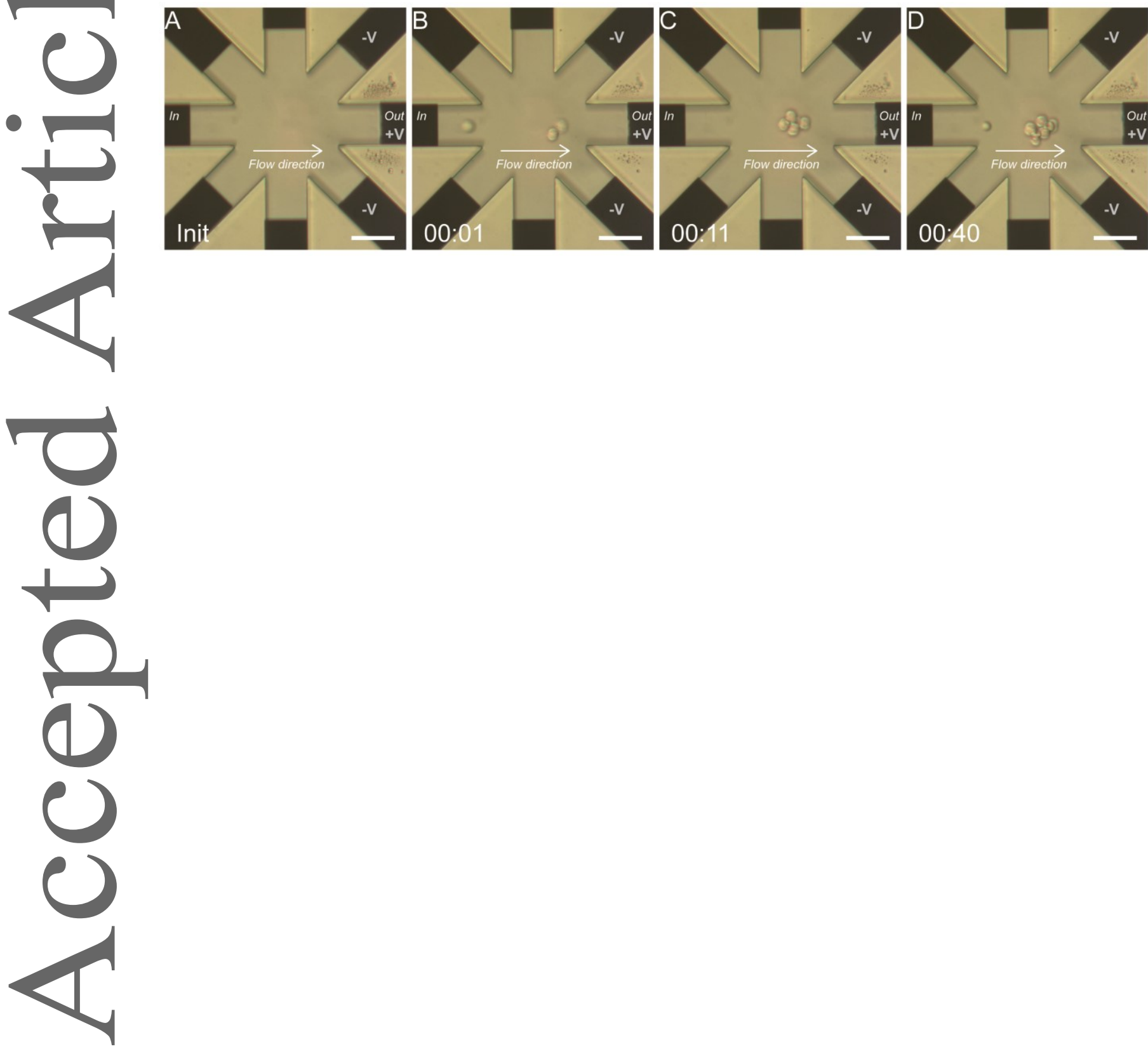

This article is protected by copyright. All rights reserved. 
Figure 4 A) COMSOL Multiphysics 2D Simulation of the fluid velocity profile, in $\mu \mathrm{m} / \mathrm{s}$, in the improved chip design with an inlet velocity of $500 \mu \mathrm{m} / \mathrm{s}$. B) 2D simulation of the trapping position of a cell using 3 electrodes supplied with $V=10 V_{p}$ at $10 \mathrm{kHz}$. The surface represents $\frac{\partial E^{2}}{\partial x}$. The white dot indicates the trapping position, $x_{\text {trap }}$, of a HEK cell (diameter 15 $\mu \mathrm{m})$. C) Evolution of the DEP and drag forces along the BB' cut. The resulting force is represented with the black dashed line and the red dashed vertical lines indicate the position where the resulting force is equal to zero. The gray dot with the arrow indicates the direction of the resulting force on the particle and the red dashed line between zones 1 and 2 the trapping position. D) Top 3D representation of the amplitudes of the DEP (in blue) and drag (in green) forces along the $y$ axis in the chip. The white/red dashed line indicates where the $x$ component of the two forces are equal in the trapping chamber. The electrode numbers are indicated. E) Evolution of the DEP and drag forces along the $y$ axis at ) $x=x_{\text {trap }}=17 \mu \mathrm{m}$. The resulting force is represented with the black dotted line and the red dotted line indicates the positions where the resulting force is equal to zero. The gray dot with the arrow indicates the direction of the force on the particle. F) Top 3D representation of the amplitudes of the DEP (in blue) and drag (in green) forces along the $y$ axis in the chip. The white/red dashed line indicates where the $x$ components of the two forces are equal (from D) and the white cross the final position of the particle. The electrode numbers are indicated. A logarithmic scale is used for representations $D$ and $F$.
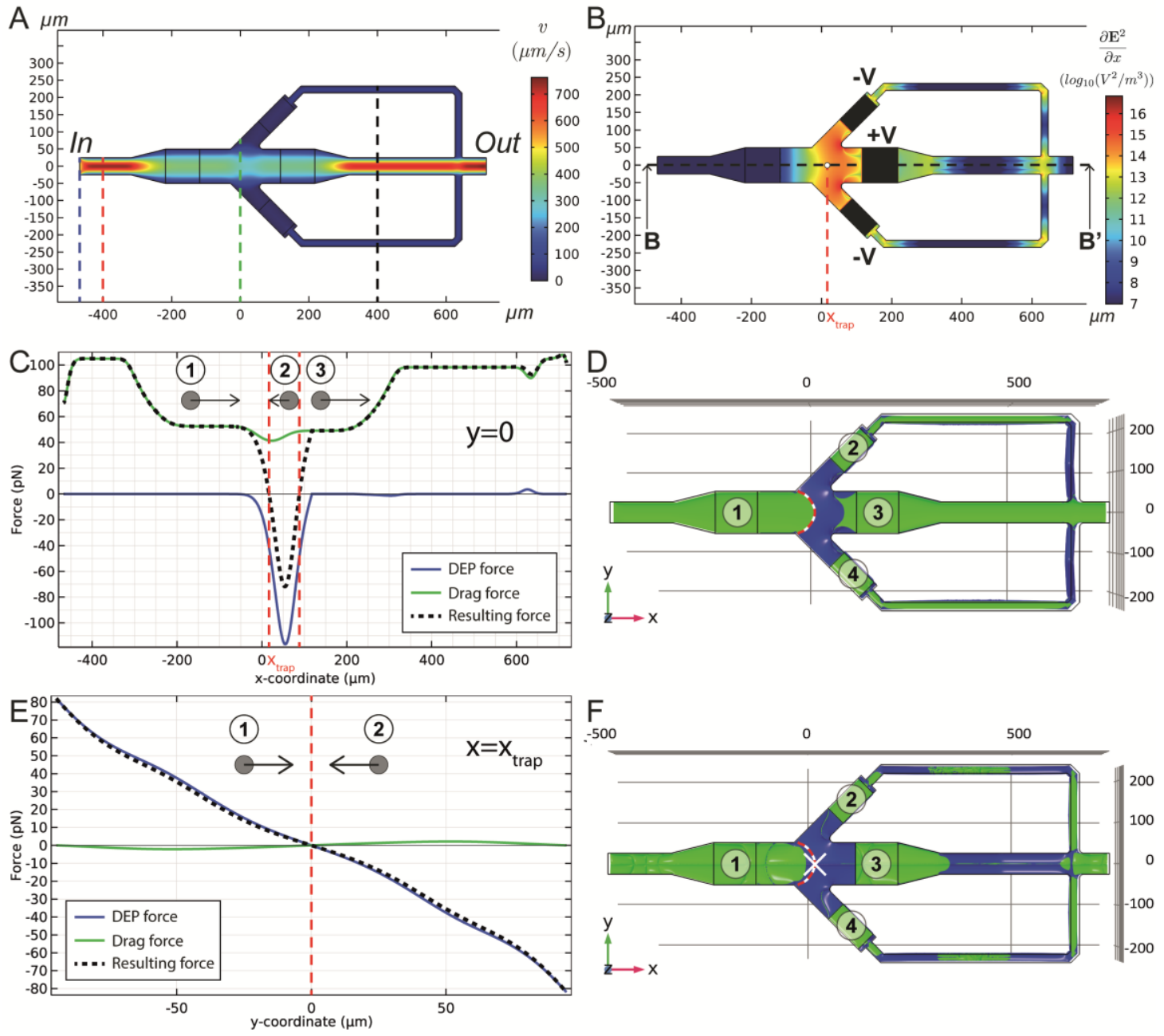

This article is protected by copyright. All rights reserved. 
